\title{
THE UNIFORM BOUND PROBLEM FOR LOCAL BIRATIONAL NONSINGULAR MORPHISMS
}

\author{
BERNARD JOHNSTON
}

\begin{abstract}
It is known that any factorization of a local birational morphism $f: \operatorname{Spec} S \rightarrow \operatorname{Spec} R$ of nonsingular (affine) schemes of arbitrary dimension via other nonsingular schemes must be finite in length. This fact generalizes the classical Local Factorization Theorem of Zariski and Abhyankar, which states that there is a unique such factorization, that given by quadratic transformations, in the surface case. A much stronger generalization is given here, namely, that there exists a uniform bound on the lengths of all such factorizations, provided that $R$ is excellent. This bound is explicitly calculated for some concrete extensions and examples are given to show that this is the strongest generalization possible in some sense.
\end{abstract}

\section{INTRODUCTION}

One salient feature of Zariski's proof of the factorization of a birational morphism $f: X^{\prime} \rightarrow X$ of nonsingular surfaces by means of a finite sequence of quadratic transformations is his reduction to the case of local morphisms (cf. [Z, Theorems II.1.1, II.1.2]). Indeed, local factorization by means of quadratic transformations is known to be possible, and to occur in a unique way, for arbitrary 2-dimensional schemes [A, Theorem 3]. In higher dimensions, unfortunately, there is no such factorization, in general, of local birational nonsingular morphisms; nor even one by means of monoidal transformations along nonsingular subvarieties [Sa, Corollary 4.5 and Sh, Example 3.2]. However, although it is necessary in higher dimensions to factor such local morphisms by blowing up singular subvarieties, it is known on the positive side that every such factorization must be finite in length [J-1, Corollary 4.10].

It is the main result of the present paper that the strongest possible such finiteness condition, namely, a uniform bound on the length of all possible local factorizations, holds for a large class of regular schemes, including the excellent ones. The statement can be made algebraically precise as follows: if $R$ is an excellent $d$-dimensional regular local ring (RLR for short) with $d \geq 2$,

Received by the editors December 30, 1987.

1980 Mathematics Subject Classification (1985 Revision). Primary 14E40; Secondary 13H05, 13B02, 13N05, 14E05.

Key words and phrases. Birational, regular local ring, excellent, quadratic transformation, monoidal transformation, Jacobian ideal, Fitting ideal, Kähler different.

Partially supported by a grant from the National Science Foundation. 
and $R \subseteq S \subseteq$ q.f. $(R)$ (the quotient field of $R$ ), where $S$ is a $d$-dimensional RLR, then there exists a positive integer $N$, depending only on $S$ and $R$, such that every chain of distinct RLRs between $S$ and $R$ has length less than or equal to $N$. There also exists such a uniform bound if a less stringent but more technical assumption than excellence is made on $R$ (Theorem 1.7). It does not seem to be known, at present, whether this technical assumption is satisfied by all RLRs.

This result is partially motivated by an analogous result of Hironaka's for projective morphisms of normal varieties: if $K$ is an algebraic function field extension of the field $k$, if $V$ is an algebraic model of $K / k$ (cf. [Z-S, pp. $115-116]$ ), and if $V^{\prime}$ is a normal algebraic model of $K / k$, then there exists an integer $N$ depending only on $V^{\prime}$ and $V$ such that any chain of successively dominating normal models of $K / k$, dominated by $V^{\prime}$, and projective over $V$, has length at most $N$ [H, Chapter II, The Main Theorem II, p. 136]. Since geometric local rings are excellent, our result gives, in particular, a local version of Hironaka's theorem. On the other hand, there is an interesting contrast here between local and global factorization: in the normal local case there exists neither a uniform bound nor even finiteness. There can exist both infinite strictly ascending and infinite strictly descending dominating chains of normal local rings already between a 2-dimensional RLR and its first quadratic transform [J-1, Examples 5.2 and 4.9].

Our local uniform bound is obtained as an easy corollary of the theory of Jacobian ideals due to Lipman and Sathaye [L-S, $\S 1$, based in turn upon a previous construction due to $\mathrm{Kunz}[\mathrm{K}, \S 1]$. $\S 1$ of the present paper is devoted to an explication of this theory and proofs of the main results. This uniform bound is often easily calculable; as a result we are able to recover as applications some previous results on minimal extensions of RLRs due to Sally [Sa, Theorem 5.1] and Shannon [Sh, Proposition 3.7]. Furthermore we obtain a considerable generalization of their results. $\S 2$ is devoted to this and to an example to show that our uniform bound is in general sufficient but not necessary for minimality.

In $\S 3$ we further explore possible finiteness conditions on the factorizations of a nonsingular birational local morphism between a fixed pair of regular affine schemes. We find that in higher dimensions there usually exist infinitely many different factorizations (although they are uniformly bounded in length) and that, moreover, their lengths can differ arbitrarily, within the uniform bound.

We adopt the conventions and notation of [J-1] throughout. In particular, all rings are assumed to be commutative with identity element. We mention a few terminological idiosyncracies. If the quasilocal (meaning unique maximal ideal) domain $(S, \mathfrak{N})$ dominates the quasilocal domain $(R, \mathfrak{M})$, then $\operatorname{restrdeg}_{R} S$ is used to denote the transcendence degree of the field $S / \mathfrak{N}$ over the canonical image of the field $R / \mathfrak{M}$. By a spot over a ring $R$ is meant a localization at a prime ideal of a finitely generated $R$-algebra. We use a special symbol, $S>R$, to indicate compactly that the quasilocal domain $S$ birationally dominates the 
quasilocal domain $R$. Finally, if $(S, \mathfrak{N})$ is a $R L R$, $\operatorname{ord}_{S}$ is used to denote the $\mathfrak{N}$-adic order valuation of q.f. $(S)$.

\section{A UNIFORM BOUND VIA JACOBIAN IDEALS}

In order to prove our main result we make use of some notions due to Kunz, and to Lipman and Sathaye (cf. [K, $\$ 1$ and L-S, §1]). The underlying idea is simply that, given a sequence of RLRs, $S \supseteq S_{1} \supseteq \cdots \supseteq S_{m} \supseteq R$, it is sometimes possible to find a single element, $b$, of $S$ such that each $S_{i}$ gives rise to a distinct factor of $b$. We recall the theory here for the convenience of the reader.

Let $S$ be a ring and let $M$ be a finite $S$-module. If $S^{\Lambda} \stackrel{U}{\rightarrow} S^{n} \rightarrow M \rightarrow 0$ is the beginning of a free resolution of $M$ over $S$, then $I_{n}(U)$ (the ideal generated in $S$ by the $n \times n$ minors of the matrix $U$ ) is called the zeroeth Fitting ideal of $M$. Fitting [F, Hauptsatz, p. 197] showed that this ideal is independent of the choice of resolution of $M$.

Now if $S$ is a spot over $R$ we define the Jacobian ideal of $S$ over $R$ (also called the zeroeth Kähler different) to be the zeroeth Fitting ideal of $\Omega_{S / R}$, the module of relative Kähler differentials of $S$ over $R$. By assumption we may write $S=A_{P}$, where $P \in \operatorname{Spec} A, A=R\left[a_{1}, \ldots, a_{n}\right]$. Since $\Omega_{S / R}=\left(\Omega_{A / R}\right)_{P}$ and since $\Omega_{A / R}$ is generated as an $A$-module by $\left\{d a_{i} \mid i=1, \ldots, n\right\}[\mathrm{M}-2, \S 25]$, $\Omega_{S / R}$ is a finite $S$-module and its zeroeth Fitting ideal is defined. We denote the Jacobian ideal by $J_{S / R}$.

In order to understand this ideal better, we assume $R$ to be Noetherian, and present $A$ :

$$
0 \rightarrow K \rightarrow R\left[X_{1}, \ldots, X_{n}\right] \stackrel{\phi}{\rightarrow} A=R\left[a_{1}, \ldots, a_{n}\right] \rightarrow 0
$$

$\left(\phi\left(X_{i}\right):=a_{i}\right)$, and apply the second fundamental exact sequence for Kähler differentials [M-2, Theorem 25.2]. Letting the generators of $K$ be $f_{1}, \ldots, f_{m} \in$ $R\left[X_{1}, \ldots, X_{n}\right]$, we conclude that $\Omega_{A / R}=\left(\Omega_{R\left[X_{1}, \ldots, X_{n}\right] / R} \otimes A\right) /\left\langle d f_{1}, \ldots, d f_{m}\right\rangle$, i.e., the free module over $A$ on the symbols $\left\{d X_{i} \mid i=1, \ldots, n\right\}$ modulo the submodule generated by the $d f_{i}$ 's. Now since $d f_{i}=\sum_{j=1}^{n} \phi\left(\partial f_{i} / \partial X_{j}\right) d X_{j}$, it follows immediately from the definition that $J_{A / R}=\phi\left(I_{n}\left(\partial f_{i} / \partial X_{j}\right)\right)$, and $J_{S / R}=J_{A / R} S$.

Let us now restrict this discussion to RLRs. Recall that $\mu(M)$ denotes the minimal number of generators of the module $M$.

Proposition 1.1. If $S \supseteq R$ are $R L R S, S$ is a spot over $R$, and q.f.(S) is algebraic (necessarily finite) over q.f.(R), then $J_{S / R}$ is principal.

Proof. Present $S: 0 \rightarrow \operatorname{ker} \phi \rightarrow A_{P} \stackrel{\phi}{\rightarrow} S \rightarrow 0$, where $A:=R\left[X_{1}, \ldots, X_{n}\right]$, $P \in \operatorname{Spec} A$. Let $Q:=P \cap R . S$ and $A_{P}$ are spots over the universally catenary local domain $R_{Q}$, so the dimension formula holds [M-1, Theorem 23], i.e.,

(1) $\operatorname{dim} R_{Q}+\operatorname{trdeg}_{R_{Q}} S=\operatorname{dim} S+\operatorname{restrdeg}_{R_{Q}} S$ and

(2) $\operatorname{dim} R_{Q}+\operatorname{trdeg}_{R_{Q}} A_{P}=\operatorname{dim} A_{P}+$ restrdeg $_{R_{Q}} A_{P}$. Noting that restrdeg $R_{Q} S$ $=$ restrdeg $_{R_{Q}} A_{P}$, we subtract (1) from (2) in order to conclude: 
(3) $n-0=\operatorname{trdeg} R_{R_{Q}} A_{P}-\operatorname{trdeg}_{R_{Q}} S=\operatorname{dim} A_{P}-\operatorname{dim} S=\mathrm{ht}(\operatorname{ker} \phi)$. But $S$ regular implies $\mu(\operatorname{ker} \phi)=\mathrm{ht}(\operatorname{ker} \phi)=n$. It follows that we may write $S=A_{P} /\left(f_{1}, \ldots, f_{n}\right)$, where $f_{1}, \ldots, f_{n} \in A$. Hence, by definition, $J_{S / R}=$ $\left(\phi\left(\partial\left(f_{1}, \ldots, f_{n}\right) / \partial\left(X_{1}, \ldots, X_{n}\right)\right)\right)$, a principal ideal.

Now we come to the key point, namely, the factorization of Jacobian ideals for RLRs.

Proposition 1.2 [L-S, Proof of Lemma, p. 201]. Let $S \supseteq T \supseteq R$ be RLRS with $S$ and $T$ spots over $R$, and q.f.(S) algebraic over q.f. $(R)$. Then $J_{S / R}=$ $J_{S / T}\left(J_{T / R} S\right)$.

Proof. We pick $f_{1}, \ldots, f_{n} \in R\left[X_{1}, \ldots, X_{n}\right]$ such that $T$ is a localization of $R\left[X_{1}, \ldots, X_{n}\right] /\left(f_{1}, \ldots, f_{n}\right)$, and pick

$$
g_{1}, \ldots, g_{m} \in R\left[X_{1}, \ldots, X_{n}, Y_{1}, \ldots, Y_{m}\right]
$$

such that $S$ is a localization of $T\left[Y_{1}, \ldots, Y_{m}\right] /\left(g_{1}, \ldots, g_{m}\right)$, using the proof of Proposition 1.1; then $S$ is a localization of

$$
R\left[X_{1}, \ldots, X_{n}, Y_{1}, \ldots, Y_{m}\right] /\left(f_{1}, \ldots, f_{n}, g_{1}, \ldots, g_{m}\right) .
$$

Modulo the appropriate relations, the Jacobian matrix of

$$
\partial\left(f_{1}, \ldots, f_{n}, g_{1}, \ldots, g_{m}\right) / \partial\left(X_{1}, \ldots, X_{m}, Y_{1}, \ldots, Y_{m}\right)
$$

appears with the Jacobian matrices of $\partial\left(f_{1}, \ldots, f_{n}\right) / \partial\left(X_{1}, \ldots, X_{n}\right)$ and of $\partial\left(g_{1}, \ldots, g_{m}\right) / \partial\left(Y_{1}, \ldots, Y_{m}\right)$ along its main diagonal, with zeroes elsewhere. The result now follows from elementary facts of determinants.

In order to obtain a uniform bound from this result, we must check that $J_{S / R} \neq(0), S$. By taking a minimal resolution of $\Omega_{S / R}$, we see that $J_{S / R}=S$ if and only if $\Omega_{S / R}=0$. The meaning of this latter condition was investigated by Kunz.

Theorem 1.3. Let $R$ be a ring, let $(S, \mathfrak{N})$ be a quasilocal ring containing $R$ such that $\Omega_{S / R}$ is $S$-finite. Let $k^{\prime}:=S / \mathfrak{N}, k:=q . f .(R /(\mathfrak{N} \cap R)), P:=\left(\mathfrak{N} \cap R\left[S^{[p]}\right]\right) S$, where $p$ is the characteristic of $S / \mathfrak{N}, S^{[p]}:=\left\{s^{p} \mid s \in S\right\}$ if $p>0$, and $S^{[p]}:=$ $\{1\}$ if $p=0$. Then:

(1) [K, Rangsatz, p. 171] $\mu\left(\Omega_{S / R}\right)=\mu\left(\Omega_{k^{\prime} / k}\right)+\mu\left(\mathfrak{N} /\left(P+\mathfrak{N}^{2}\right)\right)$, the latter two being necessarily finite; and

(2) [K, Anmerkung 1, p. 174] If $k^{\prime} / k$ is separable we can replace the last term in (1) with $\mu\left(\mathfrak{N} /(\mathfrak{N} \cap R) S+\mathfrak{N}^{2}\right)$.

Corollary 1.4. With the notation and assumptions as in Theorem 1.3, if we assume in addition that $S$ is Noetherian and that $k^{\prime}$ is a finitely generated field extension of $k$, then $J_{S / R}=S$ if and only if $k^{\prime} / k$ is (finite) separable algebraic and $\mathfrak{N}=(\mathfrak{N} \cap R) S$.

Proof. We have seen that $J_{S / R}=S$ if and only if $\Omega_{S / R}=0$. By Theorem 1.3(1) this occurs if and only if $\Omega_{k^{\prime} / k}=0$ and $\mathfrak{N}=P+\mathfrak{N}^{2}$. But, $k^{\prime}$ being 
finitely generated over $k$, the statement that $\Omega_{k^{\prime} / k}=0$ is equivalent to the statement that $k^{\prime}$ is (finite) separable algebraic over $k$ [M-1, Theorem 59(iii)]. Hence we may apply Theorem $1.3(2)$ to conclude that $\mathfrak{N}=(\mathfrak{N} \cap R) S+\mathfrak{N}^{2}$ and the Noetherian condition on $S$ now yields the result.

Corollary 1.5. If $(S, \mathfrak{N})>(R, \mathfrak{M})$ are d-dimensional local domains with $R$ normal and analytically irreducible, then $J_{S / R}=S$ if and only if $S=R$.

Proof. If $S=R$ then clearly $\Omega_{S / R}=0$ and $J_{S / R}=S$. Conversely, since $k^{\prime}=S / \mathfrak{N}$ is finite over $k=R / \mathfrak{M}=R /(\mathfrak{N} \cap R)$ in this situation [J-1, Lemma 4.5], Corollary 1.4 immediately yields that $\mathfrak{N}=\mathfrak{M S}$ and Zariski's Main Theorem [N, (37.4)] implies that $S=R$.

On the other hand, the question of when $J_{S / R}=(0)$ can be answered as follows:

Theorem 1.6 [K, Satz 4]. If $S$ is a quasilocal integral domain containing $R$, with respective quotient fields $L$ and $K$, and if $\Omega_{S / R}$ is $S$-finite, then $J_{S / R}=(0)$ if and only if $\mu\left(\Omega_{L / K}\right)>0$.

As an immediate consequence of this theorem we conclude that $J_{S / R} \neq(0)$ whenever $L$ is finite separable algebraic over $K$. This holds in particular if $S$ and $R$ are birational. Putting all of these results together now leads to our main result.

Theorem 1.7 (Uniform Bound). Assume that $(S, \mathfrak{N})>(R, \mathfrak{M})$ are d-dimensional RLRs having the property that whenever $T$ is a $R L R$ with $S \supseteq T \supseteq R$ then $T$ is a spot over $R$. Then there exists a positive integer $N$ depending only on $S$ and $R$ so that whenever $S>S_{1}>\cdots>S_{m}>R$ is a strictly decreasing chain of $R L R s$ we have $m \leq N$.

Proof. Let $b$ be a generator of the principal ideal $J_{S / R} \cdot b \neq 0$ by Theorem 1.6. Factor $b$ in the unique factorization domain (UFD) $S$ as $b=p_{1} \ldots p_{N+1}$, where $p_{i}$ is a height one prime of $S$. (We do not necessarily assume that these primes are distinct.) By iterated use of Proposition 1.2, we conclude that $J_{S / R}=J_{S / S_{1}}\left(J_{S_{1} / S_{2}} \cdots J_{S_{m} / R}\right) S$. All of these are principal ideals in $S$ and not equal to $S$ by Corollary 1.5 , since the chain is strictly decreasing. It follows that $m \leq N$.

Remark 1.8. If $J_{S / R}=b S$, the uniform bound given above is the number of irreducible factors (counting multiples) of $b$ in the UFD $S$, minus 1 . Since this latter number is less than or equal to $\operatorname{ord}_{\mathfrak{N}}\left(J_{S / R}\right)-1$, Theorem 1.7 also implies that $\operatorname{ord}_{\mathfrak{N}}\left(J_{S / R}\right)-1$ is a (perhaps more easily calculable) uniform bound.

It is important to note that the hypotheses of the theorem are fulfilled for a very large class of RLR's, namely the excellent ones. More generally, we can use the following definition, due to Heinzer, Huneke, and Sally.

Definition 1.9. A $d$-dimensional local domain $R$ is said to have property $\bar{L}$ iff every $d$-dimensional normal spot birationally dominating $R$ is analytically normal. 
Corollary 1.10. If $S>R$ are d-dimensional $R L R$ s and $R$ has property $\bar{L}$, then there exists a uniform bound on chains of RLRs between $S$ and $R$, in the sense of Theorem 1.7.

Proof. We need only see that every RLR $T$ with $S>T>R$ is a spot over $R$, in order to apply Theorem 1.7. Since $T$ and $R$ are RLRs they are normal and analytically unramified and we simply quote [H-H-S, Theorem 1], which now guarantees that $T$ is a spot over $R$.

Recall that excellent local domains [M-1, Theorem 79] and 2-dimensional RLRs [L, Proposition, p. 160] always satisfy property $\bar{L}$. We immediately get the significant corollary.

Corollary 1.11. If $S>R$ are d-dimensional $R L R s$ with either $R$ excellent or $d=2$, then there exists a uniform bound on chains of RLRs between $S$ and $R$ as above.

Notice that this partially recovers the Local Factorization Theorem of Zariski and Abhyankar, which states that in the 2-dimensional case there is a unique finite sequence between $S$ and $R$, with each successive step a quadratic transform of the previous step.

It is apparently still an open question as to whether an arbitrary RLR $R$ has the property that every RLR of the same dimension birationally dominating $R$ is a spot over $R$. This at least holds for "geometric" RLRs (i.e., spots over a field), for complete RLRs, and for RLRs containing a field of characteristic 0 which satisfy the "weak Jacobian condition," as these are all excellent [M-1, (34.A) and Theorem 101]. One should note that there do exist examples of nonexcellent RLRs, for example in [M-1, (34.B) and R, $\S \S 1,4]$.

\section{ApPlications AND EXAMPLES}

Let us begin by explicitly calculating the uniform bound produced in the previous section. This will allow us to recover some previously known results due to Sally and Shannon on the minimality of certain extensions of RLRs. For simplicity we consistently assume that our ground RLR is excellent in this section, although, as is clear from the previous section, somewhat more general hypotheses could be used.

Example 2.1. Let $R$ be an excellent RLR and let $S:=R[a / b]_{\mathfrak{N}}$ be a RLR with $\mathfrak{N} \in \operatorname{Spec}(R[a / b])$ such that $\mathfrak{M} \subseteq \mathfrak{N}$. Assume that $a$ and $b$ are relatively prime in $R$. Then $J_{S / R}=b S$, for if we present $S: 0 \rightarrow \operatorname{ker} \phi \rightarrow R[Z]_{\mathfrak{N}^{\prime}} \stackrel{\phi}{\rightarrow}$ $S \rightarrow 0$, with $\mathfrak{N}^{\prime}$ the preimage of $\mathfrak{N}$, then $\operatorname{ker} \phi=(b Z-a)$, as $a, b$ is an $R$-sequence [D, Proposition 2]. It follows that just the number of irreducible factors of the denominator is a uniform bound in this case. More concretely, if $x, y$ form part of a regular system of parameters in $R$, and if $N \geq 1$, then $R\left[y / x^{N+1}\right]_{\left(\mathfrak{M}, y / x^{N+1}\right)}=(S, \mathfrak{N})$ is a RLR of the type described. In this context, the uniform bound constructed in Theorem 1.7 is $N$ and this bound is actually 
attained, because by setting $S_{i}:=R\left[y / x^{N-i+1}\right]_{\mathfrak{N} \cap R\left[y / x^{N-i+1}\right]}$, we get a strictly decreasing chain: $S>S_{1}>\cdots>S_{N}>R$.

Now if $S=R[1 / b]_{\mathfrak{N}}$ as in the example above, and if $b$ is irreducible in $S$, then one can immediately conclude that there are no RLRs properly between $S$ and $R$, i.e., $S$ is a minimal extension of $R$ as a RLR. This then easily recovers, if $R$ is excellent, the following result found by Sally [Sa, Theorem 5.1] and also partially found by Shannon [Sh, Proposition 3.7].

Corollary 2.2. (1) If $R$ is an excellent $R L R$ and $S$ is a simple monoidal transform of $R$, then $S$ is a minimal extension of $R$ as a $R L R$.

(2) If $(R, \mathfrak{M})$ is an excellent $R L R$ of dimension larger than 2 and if $S$ is a Sally extension of $R$ (i.e., an extension $S=R[a / b]_{\mathfrak{N}}$ where $\mathfrak{M} \subseteq \mathfrak{N}, a$ and $b$ are relatively prime in $R,(a, b) R$ is prime, $a, b \neq 0, a \in \mathfrak{M} \backslash \mathfrak{M}^{2}$, and $\left.b \in \mathfrak{M}^{2}\right)$, then $S$ is minimal over $R$ as a $R L R$.

With the tools at hand, it is now possible to strengthen this result with respect to other familiar examples of birational extensions of RLRs.

Proposition 2.3. Let $R$ be an excellent $R L R$, and let $S$ be a monoidal transform of $R$ with nonsingular center $P$. If ht $P=n$, then there exist at most $n-2$ distinct $R L R$ s in any chain between $S$ and $R$. Moreover, there exists $a$ chain of length exactly $n-2$ between $S$ and $R$.

Proof. By assumption, we may write $S=R\left[x_{2} / x, x_{3} / x, \ldots, x_{n} / x\right]_{\mathfrak{N}}$, where $\mathfrak{N}$ is a height $d$ prime ideal of $R\left[x_{2} / x, x_{3} / x, \ldots, x_{n} / x\right] \quad(d:=\operatorname{dim} R)$ and $\left(x, x_{2}, \ldots, x_{n}\right)=P$ is a regular prime (i.e., $R / P$ is a $\left.\operatorname{RLR}\right)$. As $x, x_{2}, \ldots, x_{n}$ forms an $R$-sequence, we can again apply [D, Proposition 2] in order to conclude that if $\phi: R\left[W_{2}, \ldots, W_{n}\right]_{\mathfrak{N}^{\prime}} \rightarrow S$, then $\operatorname{ker} \phi=\left(x W_{2}-x_{2}, \ldots, x W_{n}-\right.$ $x_{n}$ ). Thus the Jacobian ideal is generated by the determinant of the $n-1$ by $n-1$ matrix which has $x$ in each position along the main diagonal and zeroes elsewhere, so $J_{S / R}=\left(x^{n-1}\right) S$. Since $x$ is irreducible in $S$, the bound follows immediately from Corollary 1.11 .

In this extension, if

$$
R_{i}:=R\left[\frac{x_{2}}{x}, \frac{x_{3}}{x}, \ldots, \frac{x_{j+1}}{x}\right]_{\mathfrak{N} \cap R\left[x_{2} / x, x_{3} / x, \ldots, x_{i+1} / x\right]}
$$

then $R<R_{1}<\cdots<R_{n-1}=S$ forms a chain of RLRs actually attaining the uniform bound.

Observing the examples above, it is natural to wonder whether the uniform bound given in Corollary 1.11 is always attained. This is unfortunately not the case, as is evident from the following example.

Example 2.4. Let $(R, \mathfrak{M}):=k\left[X Y Z, Y^{2} Z, X^{3} Z\right]_{\left(X Y Z, Y^{2} Z, X^{3} Z\right)}$, and set $S:=$ $k[X, Y, Z]_{(X, Y, Z)}$, where $k$ is any field, and $X, Y$, and $Z$ are indeterminates. 
Since the matrix of exponents here is

$$
\left(\begin{array}{lll}
1 & 1 & 1 \\
0 & 2 & 1 \\
3 & 0 & 1
\end{array}\right),
$$

with determinant -1 , we have $S>R$; and since $S$ is clearly a 3-dimensional RLR, it follows that $R$ is 3-dimensional (for example, from the dimension formula) and hence $R$ is an excellent RLR. We may now present $S$ as: $R\left[W_{1}, W_{2}, W_{3}\right]_{\left(\mathfrak{M}, W_{1}, W_{2}, W_{3}\right)} \stackrel{\phi}{\rightarrow} S$, and $\operatorname{ker} \phi=\left(X Y Z-W_{1} W_{2} W_{3}, Y^{2} Z-\right.$ $\left.W_{2}^{2} W_{3}, X^{3} Z-W_{1}^{3} W_{3}\right)$. Computing the Jacobian ideal, we conclude that $J_{S / R}=$ $\left(X^{3} Y^{2} Z^{2}\right) S$, so the uniform bound calculated is 7 here. However, it is known that in this particular example $S$ is minimal over $R$ as a RLR [J-2, Example 5.4].

This example shows that assuming $J_{S / R}$ to be a prime ideal of $S$ is sufficient but not necessary in order for $S>R$ to be a minimal extension of $d$-dimensional RLRs. If $S>R$ are 2-dimensional RLRs, then the condition that $J_{S / R}$ be a prime ideal is necessary and sufficient by the Local Factorization Theorem of Zariski and Abhyankar. Finding necessary and sufficient conditions for $S>R$ to be a minimal extension of $d$-dimensional RLRs, $d>2$, remains perhaps the most important and intractable question in this subject.

\section{FURTHER STRUCTURE}

Investigation shows that the structure of the partially ordered (under domination) set (poset for short) of RLRs between a fixed pair $S>R$ of higherdimensional RLRs is utterly different from that of 2-dimensional RLRs. In the 2-dimensional case, according to the Local Factorization Theorem of Zariski and Abhyankar, there exists a unique fixed chain of RLRs between $S$ and $R$. We might then say that the "height" of the poset is $N$, the length of the chain, and the "width" of the poset is 1 . Corollary 1.11 states that in higher dimensions the height of the entire poset continues to be finite ( $R$ excellent). On the other hand, once the dimension is larger than 2 , the width of the poset may become infinite in even the simplest cases.

Proposition 3.1. If $(R, \mathfrak{M})$ is a d-dimensional $R L R, d \geq 3$, and if $S$ is a monoidal transform of $R$ with nonsingular center of height $n \geq 3$ (this is just a quadratic transform in the 3-dimensional case), then the width of the poset of $R L R s$ between $S$ and $R$ is infinite.

Proof. We write $S=R\left[x_{2} / x, \ldots, x_{n-1} / x\right] \mathfrak{N}$, where ht $\mathfrak{N}=d$. Put $T_{i}:=$ $R\left[\left(x_{2}+x_{3}^{i}\right) / x\right]_{\left(\mathfrak{N},\left(x_{2}+x_{3}^{i}\right) / 2\right)}, i>0$. Then by [Sa, Theorem 5.1 or Sh, Proposition 3.7], $T_{i}>R$ is a minimal extension of RLRs. Furthermore, $T_{i}<S$ for all $i$ and $T_{i} \subseteq T_{j}$ if and only if $i=j$. Thus there are infinitely many different chains beginning immediately above $R$. 
On the other hand, considering the length of the poset of RLRs between $S$ and $R$, where $S>R$, one might wonder whether there exists some sort of "catenary" condition on the chains. This is not the case; the first example of a pair of birational RLRs having two saturated chains of different lengths was given in dimension 3 by Shannon [Sh, Remark 3.8]. In Shannon's example, there are saturated chains of lengths 1 and 2 between $S$ and $R$; moreover $R$ is excellent, so it is possible to apply Corollary 1.11 in order to conclude that the uniform bound is 2 . Therefore there are no chains longer in this example than those Shannon exhibited. We elaborate on Shannon's example below, showing a pair of 4-dimensional RLRs with saturated chains between them of lengths $2,3,4$, and 5 . This shows that all possible lengths of saturated chains, between the minimum and the maximum allowable lengths, can occur.

Example 3.2. Let $(R, \mathfrak{M})$ be an arbitrary excellent 4-dimensional RLR (e.g., $k[W, X, Y, Z]_{(W, X, Y, Z)}, k$ any field), and set

$$
(S, \mathfrak{N}):=R[x / w, y / x, z / y]_{(\mathfrak{N}, x / w, y / x, z / y)},
$$

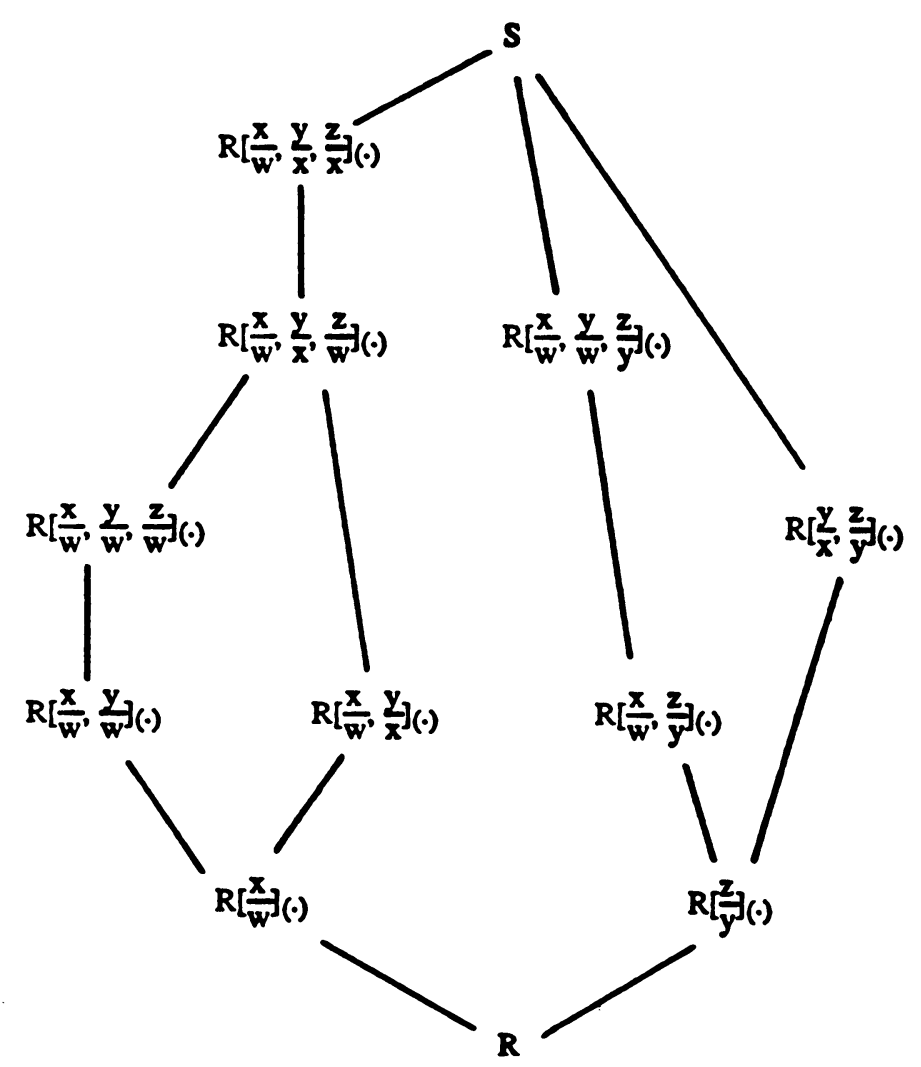

Figure 1 
where $\{w, x, y, z\}$ is a fixed regular system of parameters for $R$. For simplicity, if $T$ is a ring between $S$ and $R$ we write $T_{(\cdot)}$ in order to indicate the localization $T_{\mathfrak{N} \cap T}$. Then we have the following (see Figure 1).

Note that each of these chains, being a composition of simple monoidal transforms, is saturated by Corollary 2.2 .

If we present $S: 0 \rightarrow \operatorname{ker} \phi \rightarrow R\left[U_{1}, U_{2}, U_{3}\right] \stackrel{\phi}{\rightarrow} S \rightarrow 0$, we get $\operatorname{ker} \phi=$ $\left(x-w U_{1}, y-w U_{1} U_{2}, z-w U_{1} U_{2} U_{3}\right)$, so $J_{S / R}=\left(w^{3}(y / x)^{2} z / x\right) S$ and the uniform bound, 5 , is attained.

If $S>R$ is a minimal extension as RLRs, then we trivially have a catenary situation. The simplest case where one might have a noncatenary extension occurs when there is a saturated chain, $S>T>R$, of length 1 . We give a 3dimensional example below in order to exhibit a situation which is as far from being catenary as is possible, i.e., one in which there exists a saturated chain of length 1 and one of length $n+1$ where $n \geq 2$ is arbitrary.

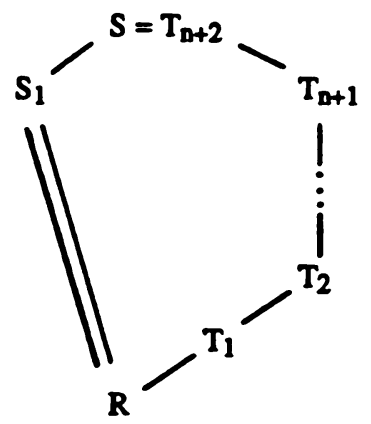

FIGURE 2

Example 3.3. Let $(R, \mathfrak{M})$ be an arbitrary 3-dimensional RLR with regular parameters $x, y$, and $z$. Let $n$ be an integer, $n>1$, set

$$
S:=R\left[\frac{y}{x}, \frac{z}{y^{n}-x^{n+1}}\right]_{\left(\mathfrak{N}, y / x, z /\left(y^{n}-x^{n+1}\right)\right)},
$$

and

$$
S_{1}:=R\left[\frac{z}{y^{n}-x^{n+1}}\right]_{\left(\mathfrak{M}, z /\left(y^{n}-x^{n+1}\right)\right)} .
$$

Going the other way, let $T_{1}:=R\left[y_{1}\right]_{\left(\mathfrak{M}, y_{1}\right)}$, where $y_{1}:=y / x$, and $T_{2}:=$ $T_{1}\left[z_{1}\right]_{\left(\mathfrak{M}, y_{1}, z_{1}\right)}$, where $z_{1}:=z /\left(y_{1}^{n}-x\right)$. Now for $3 \leq i \leq n+2$, we set $T_{i}:=$ $T_{i-1}\left[z_{1} / x^{i-2}\right]_{\left(\mathfrak{M}, y_{1}, z_{1} / x^{i-2}\right)}$. Then $S=T_{n+2}$ and all of the rings defined here are 3-dimensional RLRs birationally dominated by $S$ and birationally dominating $R$. See Figure 2. The single lines stand for simple monoidal extensions and the double line stands for a Sally extension. As both of these are known to 
be minimal extensions of RLRs, it follows that both of the chains shown are saturated.

In closing, we note that Example 3.2 gives a counterexample to the following question concerning minimal extensions of RLRs. If $S>R$, with $S$ minimal over $R$ as a RLR, and if $S^{\prime} \supseteq R^{\prime}$ are simple monoidal extensions of $S$ and $R$ respectively, then does it follow that $S^{\prime}$ is minimal over $R^{\prime}$ as a RLR? In the example above, $S_{1}$ is minimal over $R$ and $S \supseteq T_{1}$ are simple monoidal extensions of $S_{1}$ and $R$ respectively. However, not only is $S$ not minimal over $T_{1}$ as a RLR, but in fact we can construct an example with arbitrarily many RLRs between $S$ and $T_{1}$, simply by choosing $n$ to be sufficiently large.

\section{REFERENCES}

[A] S. S. Abhyankar, On the valuations centered in a local domain, Amer. J. Math. 78 (1956), 321-348.

[D] E. D. Davis, Ideals of the principal class, R-sequences and a certain monoidal transformation, Pacific J. Math. 20 (1967), 197-205.

[F] H. Fitting, Die Determinantenideale eines Moduls, Jahr. Math. -Verein. (1936).

[H] H. Hironaka, Thesis, Harvard Univ., 1960.

[H-H-S] W. Heinzer, C. Huneke, and J. D. Sally, A criterion for spots, J. Math. Kyoto Univ. 26 (1986), 667-671.

[J-1] B. Johnston, A finiteness condition on regular local overrings of a local domain, Trans. Amer. Math. Soc. 299 (1987), 513-524.

[J-2] __, Thesis, Purdue Univ., 1986.

[K] E. Kunz, Die Primidealteiler der Differenten in allgemeinen Ringen, J. Reine Angew. Math. 204 (1960), 165-182.

[L] J. Lipman, Desingularization of two-dimensional schemes, Ann. of Math. 107 (1978), 151-207.

[L-S] J. Lipman and A. Sathaye, Jacobian ideals and a theorem of Briançon-Skoda, Michigan Math. J. 28 (1981), 165-182.

[M-1] H. Matsumura, Commutative algebra (2nd ed.), Benjamin/Cummings, Reading, Mass., 1980.

[M-2] _ Commutative ring theory, Cambridge Univ. Press, Cambridge, 1986.

[N] M. Nagata, Local rings, Interscience, New York, 1962.

[R] C. Rotthaus, Nicht ausgezeichnete, universell japanische Ringe, Math. Z. 152 (1977), 107-125.

[Sa] J. D. Sally, Regular overrings of regular local rings, Trans. Amer. Math. Soc. 171 (1972), 291-300.

[Sh] D. Shannon, Monoidal transforms of regular local rings, Amer. J. Math. 95 (1973), 294-320.

[Z] O. Zariski, Introduction to the problem of minimal models in the theory of algebraic surfaces, Publ. Math. Soc. Japan, no. 4, Tokyo, 1958, pp. 1-89.

[Z-S] O. Zariski and P. Samuel, Commutative algebra, vol. II, Van Nostrand, Princeton, N.J., 1960.

Department of Mathematics, University of Utah, Salt Lake City, Utah 84112 